\title{
Formalin-Fixed Paraffin-Embedded DNA
}

National Cancer Institute

\section{Source}

National Cancer Institute. Formalin-Fixed Paraffin-Embedded DNA. NCI Thesaurus. Code C156435.

A DNA sample derived from a formalin-fixed paraffin-embedded tissue sample. 